\title{
Optimal data collection in hybrid energy-harvesting sensor networks
}

\author{
Kishor Patil ${ }^{1}$, Koen De Turck ${ }^{2}$, and Dieter Fiems ${ }^{1}$ \\ 1 Ghent University, Dept. of Telecommunications and Information Processing \\ 2 Central Supélec, Laboratoire des Signaux et Systèmes
}

\begin{abstract}
In hybrid energy harvesting sensor networks, there is a tradeoff between the cost of data collection by a wireless sink and the timeliness of the collected data. The trade-off further depends on the energy harvesting capability of the sensor nodes as sensors cannot transmit data if they do not have sufficient energy. In this paper, we propose an analytic model for assessing the value of the information that a sensor node brings to decision making. We account for the timeliness of data by discounting the value of the information at the sensor over time and adopt the energy-chunk approach (i.e. discretise the energy level) to track energy harvesting and expenditure over time. Finally, by numerical experiments, we study the optimal data collection rate for the sensor node at hand.
\end{abstract}

Keywords: Age of information; Sensor networks; Energy harvesting; Markov process.

\section{Introduction}

Wireless sensor networks (WSNs) are one of the key constituents of the Internet of Things (IoT) [1,2], and have attracted considerable research interest over the past couple of years. Sensor networks collect and monitor spatially distributed data like temperature, humidity, movement and noise [3,4], extract information from the collected data and deliver relevant information to the user. WSNs have a variety of applications including military, environmental, home and healthcare. Applications of WSNs are surveyed in [5], whereas [6] focuses on applications of WSNs in the context of the IoT.

Combining sensor networks with data analytics enable fast data-to-decision applications that act in real time on the collected data such that the value the information brings to the decision not only depends on the quality but also on the timeliness of the information. Therefore, analogous to Quality of Service which measures the performance of a data communications network, the term "Quality of Information" (QoI) has been introduced to evaluate performance of sensor networks [7-9].

The present paper investigates the QoI in energy harvesting sensor networks by calculating the value of information transmitted by energy-harvesting sensors. Energy harvesting sensor nodes mitigate their dependence on batteries by 
harvesting energy from their environment [10]. More precisely, energy harvesting sensor nodes (EHSN) use ambient sources of energy like solar, wind or heat, convert the energy into electricity which can then be used for sensing or transmitting data. Most often, the amount of energy harvested is not constant over time. Hence, energy harvesting is an additional source of uncertainty that a performance evaluation should account for. The sensor nodes under consideration operate energy neutral: all energy for sensing and transmissions is harvested, a small on-board battery providing for temporary energy storage. Summarising, the sensor nodes at hand can only transmit when the mobile sink is in range and the sensor has sufficient energy for transmitting its data.

We focus on optimal data collection, adopting the hybrid WSN of Zhou et al. [11] which consists of static sensors responsible for sensing environmental variables, and mobile sensors called IoT mobile sinks that move to designated sink locations where they gather data sensed by static sensors. Mobile sinks were introduced to overcome the hot-spot effect in sensor networks [12]. Both static and mobile sink nodes (or base terminals) collect data from sensor nodes and sometimes act as gateways to other users by processing and sending relevant information. If all sensor data is relayed by the sensor nodes to a (static) sink node, nodes closer to the static sink are more heavily loaded as they need to relay more packets to the static sink in comparison with nodes further away. As a result, they consume more energy and may die at early stage, or will frequently run out of energy if they can harvest energy. Mobile sinks overcome this problem by moving the sink around. See e.g. [13] for a discussion on design issues and challenges in existing distributed protocols for mobile sinks. Although mobility increases the network lifetime by balanced utilisation of power [14], it also introduces new challenges as delay in packet delivery should be sufficiently small [15].

The remainder of this paper is organised as follows. In the next section, we introduce a discrete-time Markov model for studying the optimal data collection probability in energy-harvesting hybrid WSNs that are unaware of the value of their information. Section 3 is then concerned with refining the model: while the sensor node is still unaware of the value of the information, it is aware whether or not there is any value. We then illustrate our approach by numerical examples in section 4 , prior to drawing conclusions in section 5 .

\section{Mathematical model}

We consider an energy harvesting sensor node. The node is equipped with a battery for storing harvested energy chunks and on-board memory to store sensed information.

We assume that time is discrete, i.e., time is divided into fixed length intervals or slots, and denote the value of the sensed data and the amount of harvested energy during slot $n$ by $S_{n}$ and $H_{n}$, respectively. The sequence $\left\{H_{n}, n \in \mathbb{N}\right\}$ constitutes a sequence of independent and identically distributed random variables, taking values in $\mathbb{N}$. Let $h_{k}$ denote the probability that $k$ energy chunks arrive 
in a slot. For further use, we always assume that the sensor node can harvest energy, that is, we assume $h_{0}<1$. Moreover, we introduce the following notation for the tail distribution function of $H_{n}$,

$$
\bar{H}_{k}=\sum_{m=k}^{\infty} h_{m}=1-\sum_{m=0}^{k-1} h_{m} .
$$

For the sequence $\left\{S_{n}, n \in \mathbb{N}\right\}$ of the value of harvested information, no independence assumptions are required. We only assume that the mean value does not depend on time; let $\bar{S}=\mathrm{E}\left[S_{n}\right]$ for $n \in \mathbb{N}$.

The battery level at the beginning of slot $n$ is denoted by $B_{n}$. We assume that transmitting data and sensing data requires $N>0$ and $M>0$ energy chunks, respectively, and that at most $C_{B}$ energy chunks can be stored in the battery. Let $T_{n}$ be the indicator that there is a transmission during slot $n$. As the sensor node harvests $H_{n}$ chunks during slot $n$, we have,

$$
B_{n+1}=\min \left(B_{n}-M 1_{\left\{B_{n} \geq M\right\}}-N T_{n}+H_{n}, C_{B}\right) .
$$

Let $V_{n}$ be the value of the sensed data in the sensor node at the beginning of the $n^{\text {th }}$ slot. We assume that the value of the sensed data is additive and discounted over time. Discounting is introduced to account for the timeliness of data, older data being less valued than recent data. Let $0 \leq \alpha \leq 1$ denote the discount factor. The values of the data at two consecutive slot boundaries then relate as,

$$
V_{n+1}=\alpha V_{n}\left(1-T_{n}\right)+S_{n} 1_{\left\{B_{n} \geq M\right\}} .
$$

The recursion above implies that any data sensed during slot $n$ cannot be transmitted during slot $n$. In addition, any chunks of energy harvested during slot $n$ cannot be used to sense and/or transmit data in slot $n$.

It now remains to express the indicator $T_{n}$ that there is a transmission in terms of $B_{n}$. To this end, let $P_{n}$ be the indicator that the data is collected from the sensor during slot $n$, that is, $P_{n}$ is 1 if data is collected at time $n$. Let $p=\mathrm{P}\left[P_{n}=1\right]=\mathrm{E}\left[P_{n}\right]$ be the collection probability, $0<p \leq 1$. We assume that the sensor node cannot evaluate the value of its information, and therefore transmits if sufficient energy is available. We therefore have,

$$
T_{n}= \begin{cases}1 & \text { for } P_{n}=1 \text { and } B_{n} \geq M+N \\ 0 & \text { otherwise }\end{cases}
$$

The set of recursions above now allows for determining the value of the information collected by the mobile sink. To this end, let $v_{k}=\mathrm{E}\left[V_{n} 1\left\{B_{n}=k\right\}\right]$ be the mean value of the information at the sensor node for battery level $k$, and let $b_{k}=\mathrm{P}\left[B_{n}=k\right]$ be the probability of having battery level $k$. In view of the equations for $V_{n}, B_{n}$ and $T_{n}$ and by conditioning on the battery level and the availability of a transmission opportunity in the preceding slot, we find that the 
mean value of the information at battery level $k$ adheres to the following set of equations,

$$
\begin{aligned}
v_{k}=\alpha \sum_{\ell=0}^{M-1} v_{\ell} h_{k-\ell}+\alpha \sum_{\ell=M}^{C_{B}} v_{\ell} h_{k-\ell+M}-\alpha p \sum_{\ell=M+N}^{C_{B}} v_{\ell} h_{k-\ell+M} \\
+\bar{S}\left(b_{k}-\sum_{\ell=0}^{M-1} b_{\ell} h_{k-\ell}\right),
\end{aligned}
$$

for $k=0,1, \ldots, C_{B}-1$, whereas for $k=C_{B}$ we have,

$$
\begin{aligned}
v_{C_{B}}=\alpha \sum_{\ell=0}^{M-1} v_{\ell} \bar{H}_{C_{B}-\ell}+\alpha \sum_{\ell=M}^{C_{B}} v_{\ell} \bar{H}_{C_{B}-\ell+M} & \alpha p \sum_{\ell=M+N}^{C_{B}} v_{\ell} \bar{H}_{C_{B}-\ell+M} \\
& +\bar{S}\left(b_{C_{B}}-\sum_{\ell=0}^{M-1} b_{\ell} \bar{H}_{C_{B}-\ell}\right) .
\end{aligned}
$$

To find the battery level probabilities, we again condition on the battery level and the availability of a transmission opportunity in the preceding slot. We have the following equations for the battery level probabilities,

$$
b_{k}=\sum_{\ell=0}^{M-1} b_{\ell} h_{k-\ell}+\sum_{l=M}^{C_{B}} b_{\ell} h_{k-\ell+M}+p \sum_{l=M+N}^{C_{B}} b_{\ell}\left(h_{k-\ell+M+N}-h_{k-\ell+M}\right),
$$

for $k=0,1, \ldots, C_{B}-1$.

The system of equations (3) along with the normalisation condition

$$
\sum_{k=0}^{C_{B}} b_{k}=1
$$

allows for solving for the probabilities $b_{k}$. We can then solve the system of equations (1) for the conditional mean values $v_{k}$.

Finally, we express the mean value of the sensed data per time slot that is actually collected in terms of the $v_{k}$ 's as follows,

$$
\bar{V}=p \sum_{k=M+N}^{C_{B}} v_{k}
$$

as there are only transmissions if the battery level exceeds the threshold and there is a transmission opportunity.

Remark 1. If $C_{B}$ is small, both systems of equations (for $b_{k}$ and $v_{k}$ ) are easily solved. For larger $C_{B}$, we can exploit structural properties of the systems of equations. As $h_{k}=0$ for $k<0$, we see that equation (3) expresses $b_{k}$ in terms of the probabilities $b_{\ell}$ for $\ell \leq k+M+N$. This implies that the Markov chain $B_{k}$ is a $G / M / 1$-type Markov chain. Hence, solution methods for a $G / M / 1$-type Markov chain (e.g. [16]) can be applied to solve the system of equations of the $b_{k}$ 's. Analogously, we see that equation (1) expresses $v_{k}$ in terms of $v_{\ell}$, for $\ell<k+M$. 
Remark 2. The assumption $h_{0}>0$ and equation (1) imply that $\lim _{p \rightarrow 0} v_{k}<\infty$ for $k<C_{B}$ and any value of $\alpha$, as well as for $k=C_{B}$ and $\alpha<1$. For $\alpha=1$, we have $0<\lim _{p \rightarrow 0} p v_{C_{B}}<\infty$. This shows that for $\alpha<1$ we have $\lim _{p \rightarrow 0} \bar{V}=0$, whereas for $\alpha=1$ we have $\lim _{p \rightarrow 0} \bar{V}=\lim _{p \rightarrow 0} p v_{C_{B}}>0$.

Remark 3. We assumed that the battery can be modelled by a queueing-like system with "arrivals" and "departures" of chunks of energy. Such battery models were considered several times in literature, see e.g. [17, 18]. While being modelled as a queueing system, one prefers that the battery/queue is full in contrast to most other queueing systems. That is, the preferred operation differs significantly.

\section{A refinement}

While it is reasonable to assume that the sensor node cannot value its data, it is not always reasonable to assume that the sensor node is unaware of the presence of data. We therefore now refine the model such that the sensor node does not transmit when there is no data to transmit. To this end, we introduce the indicator $A_{n}$ which is 1 if the node has data to transmit and 0 otherwise. Of course we have $A_{n}=1_{\left\{V_{n}>0\right\}}$, but it is more convenient to track $A_{n}$ separately, see below. In addition, we assume that the sequence $\left\{S_{n}, n \in \mathbb{N}\right\}$ constitutes a sequence of independent and identically distributed random variables with common mean $\bar{S}=\mathrm{E}\left[S_{n}\right]$ and with non-zero probability mass $s_{0}=\mathrm{P}\left[S_{n}=0\right]$ when there is no data sensed. Following similar arguments as in the preceding section, we find that the system variables $V_{n}, A_{n}$ and $B_{n}$ adhere the following set of recursive equations,

$$
\begin{aligned}
& V_{n+1}=\alpha V_{n}\left(1-T_{n}\right)+S_{n} 1_{\left\{B_{n} \geq M\right\}}, \\
& A_{n+1}=1_{\left\{S_{n}>0\right\}} 1_{\left\{B_{n} \geq M\right\}}+\left(1-1_{\left\{S_{n}>0\right\}} 1_{\left\{B_{n} \geq M\right\}}\right) A_{n}\left(1-T_{n}\right), \\
& B_{n+1}=\min \left(B_{n}-M 1_{\left\{B_{n} \geq M\right\}}-N T_{n}+H_{n}, C_{B}\right),
\end{aligned}
$$

where $T_{n}$ denotes the indicator that there is a transmission,

$$
T_{n}=1_{\left\{B_{n} \geq M+N\right\}} A_{n} P_{n} .
$$

That is, there is a transmission when (i) there is sufficient energy, (ii) there is something to send and (iii) there is a transmission opportunity.

In contrast to the preceding section, the sequence $\left\{B_{n}, n \in \mathbb{N}\right\}$ is not a Markov chain as the evolution of $B_{n}$ depends on the presence or absence of information. Therefore we focus on the sequence $\left\{\left(A_{n}, B_{n}\right), n \in \mathbb{N}\right\}$ which is a Markov chain. Let $\widetilde{b}_{k}=\mathrm{P}\left[B_{n}=k, A_{n}=0\right]$ be the probability that there are $k$ chunks of energy in the battery at slot boundaries and there is no information at the sensor node. By conditioning on the values of $P_{n}, H_{n}$ and $A_{n}$, we find,

$$
\widetilde{b}_{k}=\sum_{\ell=0}^{M-1} \widetilde{b}_{\ell} h_{k-\ell}+s_{0} \sum_{\ell=M}^{C_{B}} \widetilde{b}_{\ell} h_{k-\ell+M}+p s_{0} \sum_{\ell=M+N}^{C_{B}} \widehat{b}_{\ell} h_{k-\ell+M+N},
$$


for $k=0,1, \ldots, C_{B}-1$, and,

$$
\widetilde{b}_{C_{B}}=\sum_{\ell=0}^{M-1} \widetilde{b}_{\ell} \bar{H}_{C_{B}-\ell}+s_{0} \sum_{\ell=M}^{C_{B}} \widetilde{b}_{\ell} \bar{H}_{C_{B}-\ell+M}+p s_{0} \sum_{\ell=M+N}^{C_{B}} \widehat{b}_{\ell} \bar{H}_{C_{B}-\ell+M+N} .
$$

Here, $\widehat{b}_{k}=\mathrm{P}\left[B_{n}=k, A_{n}=1\right]$ is the probability to have $k$ chunks and information at a slot boundary. Again, by conditioning on the values of $P_{n}, H_{n}$ and $A_{n}$, we have,

$$
\begin{aligned}
\widehat{b}_{k}=\sum_{\ell=0}^{M-1} \widehat{b}_{\ell} h_{k-\ell}+\sum_{\ell=M}^{C_{B}}\left(\widehat{b}_{\ell}\right. & \left.+\widetilde{b}_{\ell}\left(1-s_{0}\right)\right) h_{k-\ell+M} \\
& +p \sum_{\ell=M+N}^{C_{B}} \widehat{b}_{\ell}\left(\left(1-s_{0}\right) h_{k-\ell+M+N}-h_{k-\ell+M}\right)
\end{aligned}
$$

for $n=0,1, \ldots, C_{B}-1$. Complementing the set of equations above with the normalisation condition,

$$
\sum_{n=0}^{C_{B}} \widehat{b}_{n}+\widetilde{b}_{n}=1
$$

allows for determining the probabilities $\widetilde{b}_{n}$ and $\widehat{b}_{n}$. For further use, we also define the probability $b_{n}$ to have $n$ chunks of energy at the node, irrespective of whether there is information at the node,

$$
b_{n}=\widetilde{b}_{n}+\widehat{b}_{n} .
$$

We now focus on the mean value of the information at the sensor node. Let $v_{n}=\mathrm{E}\left[V_{k} 1_{\left\{B_{k}=n\right\}}\right]$ be the mean value of information when there are $n$ chunks of energy available. Notice that by the definition of $A_{k}$ we have that $A_{k}=0$ implies $V_{k}=0$. Hence, there is no need to focus on the expectation of $V_{k}$ for $A_{k}=0$ and $A_{k}=1$ as $v_{n}=\mathrm{E}\left[V_{k} 1_{\left\{B_{k}=n\right\}}\right]=\mathrm{E}\left[V_{k} 1_{\left\{B_{k}=n, A_{k}=1\right\}}\right]$ and $\mathrm{E}\left[V_{k} 1_{\left\{B_{k}=n, A_{k}=0\right\}}\right]=0$. By conditioning on the presence of transmission opportunities, the availability of data and the amount of harvested energy, we find,

$$
\begin{aligned}
v_{k}=\sum_{\ell=0}^{M-1} v_{\ell} \alpha h_{k-\ell}+\sum_{\ell=M+N}^{C_{B}} \widehat{b}_{\ell} p \bar{S} h_{k-\ell+M+N} & +\sum_{\ell=M}^{C_{B}}\left(\alpha v_{\ell}+\bar{S} b_{\ell}\right) h_{k-\ell+M} \\
& -\sum_{\ell=M+N}^{C_{B}}\left(\alpha v_{\ell}+\widehat{S}_{\ell}\right) p h_{k-\ell+M},
\end{aligned}
$$

for $k=0, \ldots, C_{B}-1$, while for $k=C_{B}$ we have,

$$
\begin{array}{r}
v_{C_{B}}=\sum_{\ell=0}^{M-1} v_{\ell} \alpha \bar{H}_{C_{B}-\ell}+\sum_{\ell=M+N}^{C_{B}} \widehat{b}_{\ell} p \bar{S} \bar{H}_{C_{B}-\ell+M+N}+\sum_{\ell=M}^{C_{B}}\left(\alpha v_{\ell}+\bar{S} b_{\ell}\right) \bar{H}_{C_{B}-\ell+M} \\
-\sum_{\ell=M+N}^{C_{B}}\left(\alpha v_{\ell}+\bar{S}_{\ell}\right) p \bar{H}_{C_{B}-\ell+M} .
\end{array}
$$


Finally, as we collect information when there is energy and information at the sensor at a transmission opportunity, the mean value of the information collected at a slot boundary equals,

$$
\bar{V}=p \sum_{\ell=M+N}^{C_{B}} v_{\ell} .
$$

Remark 4. We note that the model of this section does not reduce to the model of section 2 when the sensor always picks up information, that is, for $s_{0}=0$. Even for $s_{0}=0$, it is still possible that there is no new value of information during a time slot as there may be no sensing due to a lack of energy. If we additionally assume that sensing does not take energy $(M=0)$, both models do correspond. Indeed, equation (4) then implies $\widetilde{b}_{k}=0$, whereas the sets of equations (3) and (5) are the same.

\section{Optimal data collection and numerical results}

We now investigate the optimal data collection policy for the sensor node at hand. We assume that there is a cost $c$ associated to data collection such that the average value after collection equals,

$$
\bar{V}_{p}=-c p+\bar{V} .
$$

We first illustrate the analysis of the initial model, introduced in section 2 by some numerical examples. We then complement these with some numerical results for the refinements which were discussed in section 3. In either case, we particularly focus on the optimal collection probability $p$.

\subsection{Information-agnostic transmissions}

We first investigate how the battery capacity and discount factor affect the mean value of information. To this end, we consider the initial model assuming Poisson energy harvesting - the energy harvesting distribution is Poisson with mean $\lambda$ - and energy discretisation such that $M=1$ and $N=4$ chunks of energy are required for sensing and transmitting, respectively.

Figures $1(\mathrm{a})$ and $1(\mathrm{~b})$ depict the value of information $\bar{V}_{p}$ in terms of the data collection rate $p$. We assume that the cost of collection is half the mean value of information collected in a slot: $c=1$ and $\bar{S}=2$. Figure 1(a) fixes the discount factor to $\alpha=0.9$ and shows $\bar{V}_{p}$ for various values of the battery capacity $C_{B}$ as indicated. In contrast, figure 1(b) fixes the battery capacity to $C_{B}=32$ and shows $\bar{V}_{p}$ for various values of the discount factor. For both figures, the mean number of chunks of harvest energy equals $\lambda=2$.

It can be seen from both figures that the value of information $\bar{V}_{p}$ first increases for increasing values of the collection probability and then decreases again. This observation can be explained by noting that for higher values of $p$ the chance 


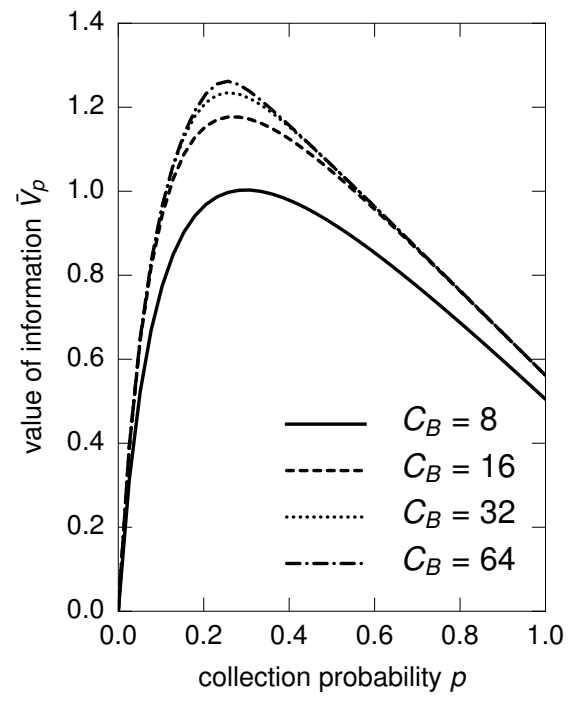

(a) Varying $C_{B}$

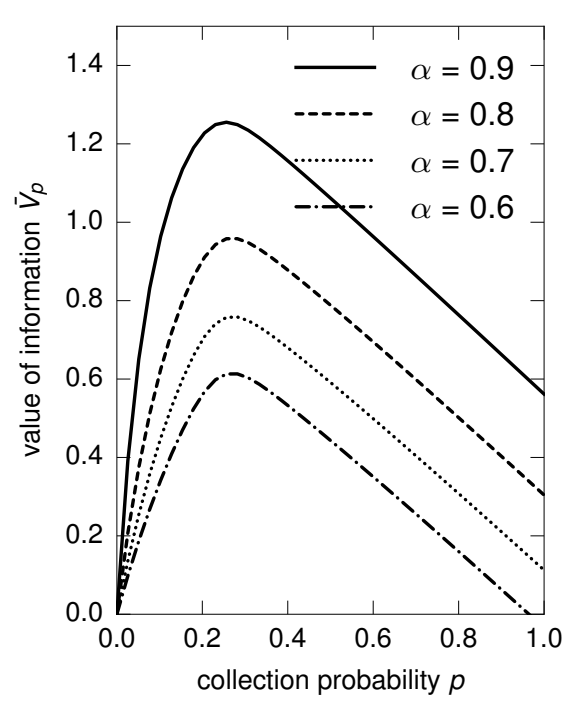

(b) Varying $\alpha$

Fig. 1. The mean value of the information $\bar{V}_{p}$ versus the polling probability $p$ for (a) different values of the battery capacity $C_{B}$ and (b) different values of the decay rate $\alpha$ as indicated.

of having insufficient energy increases as more energy is consumed for transmissions. For high $p$, it is quite likely that there is sufficient energy to transmit such that the possible gain of frequent data collection cannot compensate the collection cost. Further, figure 1(a) shows that it is beneficial to increase the battery size. Having a battery with more capacity facilitates compensating periods with little energy harvesting. However, the marginal gain obtained by increasing the battery capacity quickly disappears. Increasing the discounting factor is equally beneficial as can be seen from figure 1(b). A higher discounting factor implies that the value of information decays more slowly such that more information is available during collection.

We now focus on the effects of the distribution of the harvested energy. To this end, figures 2(a) and 2(b) depict the mean value of information versus the collection probability $p$ for Poisson distributed (figure 2(a)) and geometrically distributed (figure 2(b)) energy harvesting. Different values for the mean number $\lambda$ of harvested chunks in a slot are assumed as indicated. As for the preceding figures, $M=1$ and $N=4$ chunks of energy are required for sensing and transmitting, respectively. Moreover, the discounting factor is equal to $\alpha=0.9$, the mean value of sensed information $\bar{S}=2$ is twice the collection cost $c=1$, and the battery can store up to $C_{B}=32$ chunks of energy.

Comparing figures 2(a) and 2(b) reveals that the distribution of the harvested energy considerable affects the value of information $\bar{V}_{p}$ as well as the optimal 


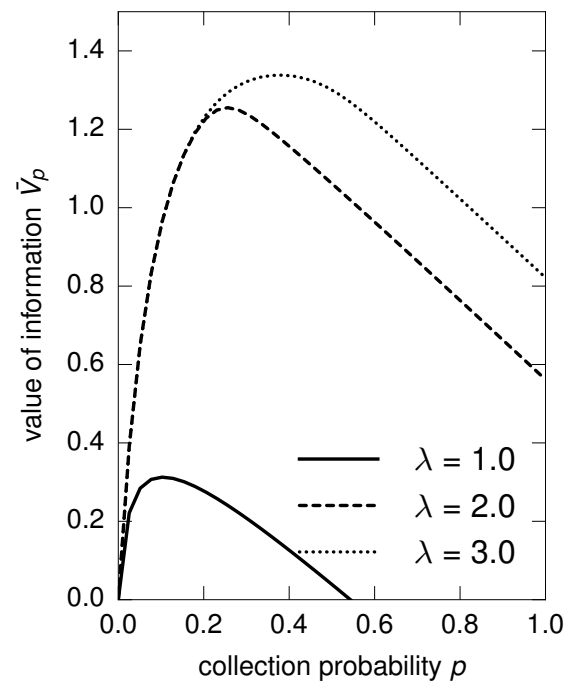

(a) Poisson

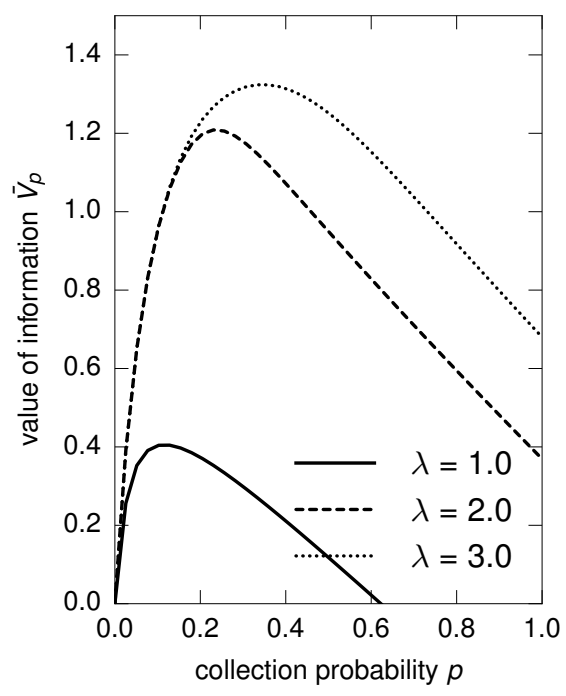

(b) Geometric

Fig. 2. The mean value of the information $\bar{V}_{p}$ versus the polling probability $p$ for a Poisson (a) and geometric (b) energy harvesting distribution, for different values of the mean amount of harvested energy $\lambda$ as indicated.

collection probability $p$. Increasing the harvesting capability of the sensor node (increasing $\lambda$ ) is initially beneficial, but the marginal gain from a further increase quickly disappears. Indeed, if there is already sufficient energy, one cannot expect that a further increase of the harvesting capability considerably improves the performance of the sensor node.

Finally, we consider the effect of the collection cost on the optimal collection probability and the associated optimal value of information. Figure 3(a) shows the optimal collection probability $p$ versus the collection cost $c$ for different values of the discount factor $\alpha$ as depicted. Figure 3(b) depicts the value of information corresponding to this optimal probability versus the collection cost $c$. Apart from the discount factor and the collection cost, the parameters are chosen as in figures 1(a) and 1(b): the mean value of sensed information equals $\bar{S}=2$, the energy harvesting distribution is a Poisson distribution with mean $1, M=1$ and $N=4$ chunks of energy are required for sensing and transmissions and the battery can store up to $C_{B}=32$ of these chunks.

The optimal collection probability quickly decreases for increasing collection costs. As the collection cost increases, any gain of collecting quickly drops due to the cost of collecting. Further, if $\alpha$ is higher, it is more beneficial to collect (see figure 1(b)) such that the optimal collection probability is higher as well. In addition, the value of information $\bar{V}_{p}$ at the optimal collection probability 


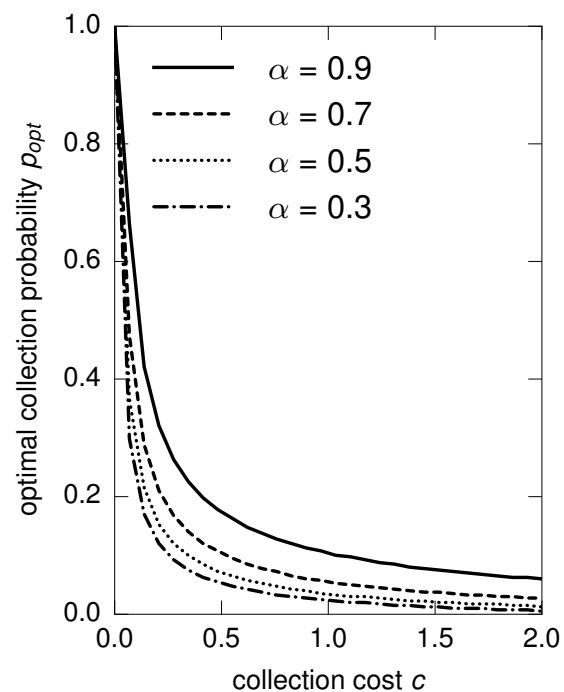

(a) optimal probability

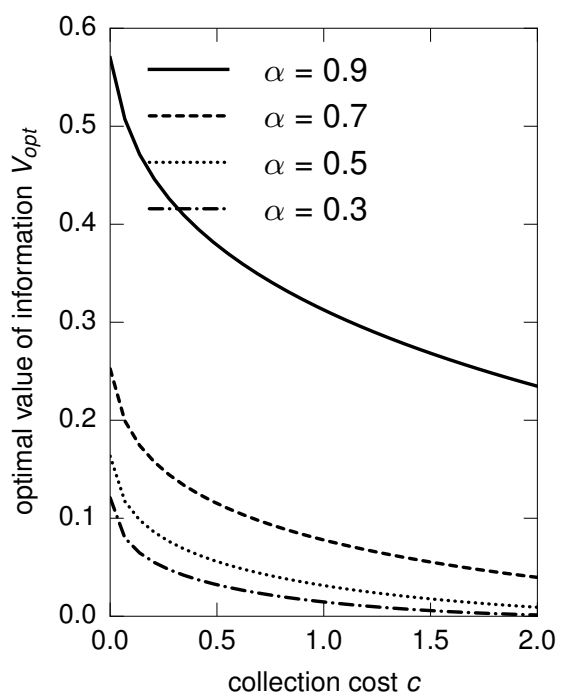

(b) optimal value

Fig. 3. The optimal collection probability $p_{\text {opt }}$ (a) and the corresponding value of information $V_{\text {opt }}$ (b) for different values of $\alpha$ as indicated.

is higher for increasing values of $\alpha$ such that it is not only beneficial to collect more, but the net gain of collecting more is higher as well.

\subsection{Information-aware transmissions}

To evaluate the use of the refined model, we now focus on how the absence of information probability $s_{0}$ affects the value of information. To make its influence clear, figures 4(a) and 4(b) depict the value of information versus the collection probability, for high and low $s_{0}$ respectively, and for various values of the discount factor $\alpha$ as depicted. To allow for a comparison with the model of section 2 and the results of section 4.1, we largely adopt the parameters of the latter: the mean value of sensed information equals $\bar{S}=2$ which is twice the cost $c=1$ of collecting. The energy harvesting distribution is a Poisson distribution with mean 2. In addition, $M=1$ and $N=4$ chunks of energy are required for sensing and transmissions and the battery can store up to $C_{B}=32$ of these chunks.

As $\bar{S}$ is fixed, a high $s_{0}$ not only means that most slots there is no information, but also means that there is considerable information in the slots with information. That is, the sensing is a bursty process. In contrast, small $s_{0}$ means that many slots carry a small amount of information. It is not surprising that these considerable differences in information arrival patterns translate into different collected values of information. This is indeed confirmed by comparing figures 4(a) and 4(b). The figures show that burstiness is beneficial. This can 


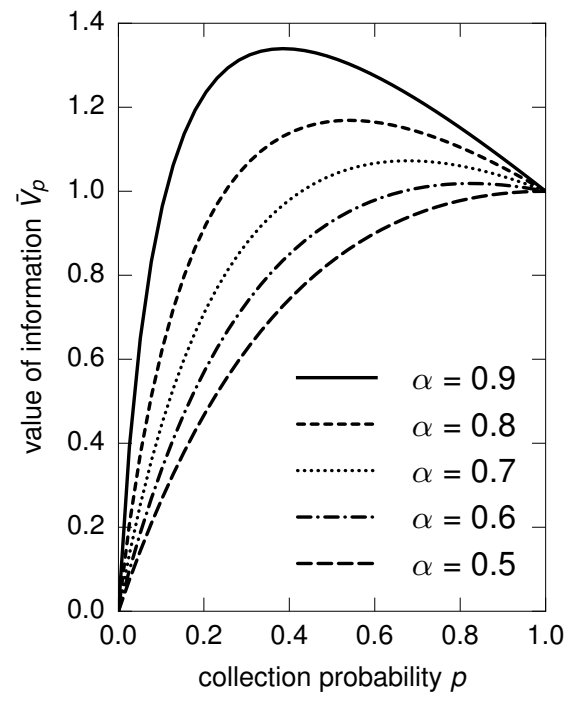

(a) $s_{0}=0.9$

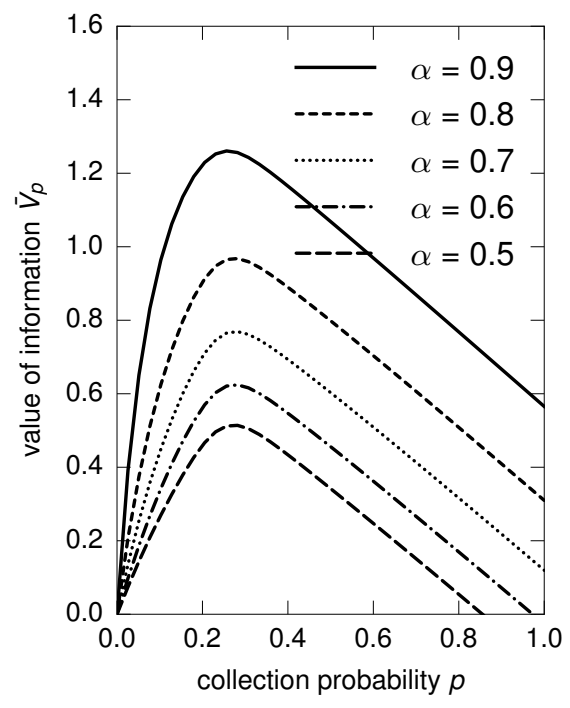

(b) $s_{0}=0.1$

Fig. 4. The mean value of information versus the collection probability for (a) $s_{0}=0.9$ and (b) $s_{0}=0.1$ and different values of $\alpha$ as indicated.

be explained by noting that no energy is lost on sending a limited amount of information. Indeed, for $s_{0}=0.9$ the chance that there is no information is considerable, whereas the value of the information is considerable whenever there is something to send. Further comparison reveals that the value of information is considerably larger for bursty sensing compared to non-bursty sensing. Moreover, the optimal collection probability is only sensitive to changes in the discount factor for bursty sensing.

This observation is also confirmed by figures $5(\mathrm{a})$ and $5(\mathrm{~b})$ which depict the optimal collection probability and the corresponding value of information versus the probability $s_{0}$, respectively. Different values of the discount factor are assumed as depicted. The same parameters are assumed as in figures 4(a) and 4(b), with the exception of $s_{0}$ which now varies. We see that the optimal collection probability and the corresponding value of information increases for increasing $s_{0}$ as explained before. Moreover, the difference between the optimal collection probabilities for different $\alpha$ is largest for high $s_{0}$. Somewhat surprising and opposite to the collection probabilities, the difference between the corresponding values of information is largest for small $s_{0}$.

\section{Conclusions}

We investigated the value of information in hybrid wireless sensor networks that harvest their energy from their environment. For energy-neutral harvesting sen- 


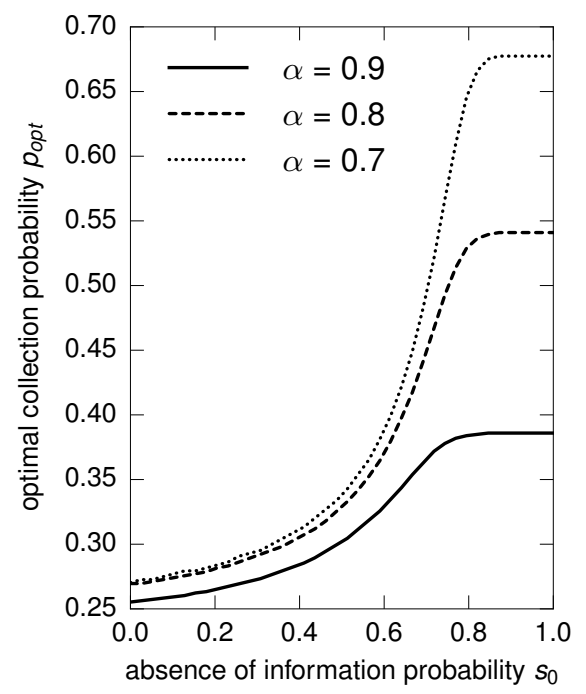

(a) optimal collection probability

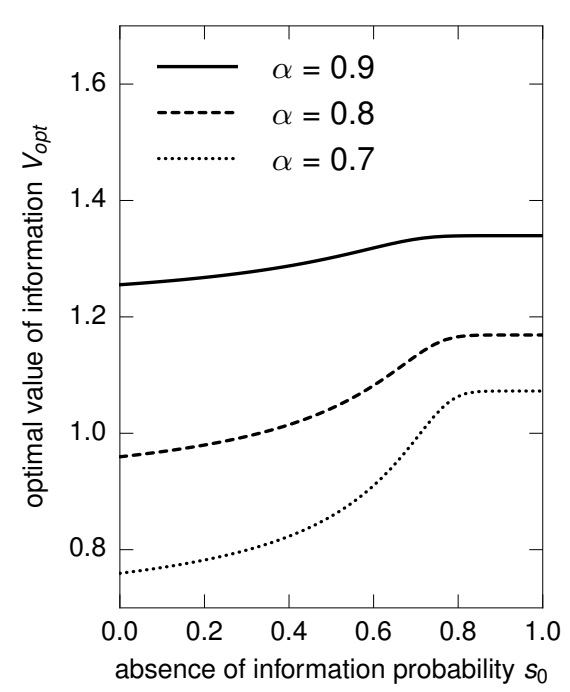

(b) optimal value of information

Fig. 5. The optimal collection probability $p_{\text {opt }}$ (a) and the value of information $V_{\text {opt }}$ (b) versus absence of information probability $s_{0}$ for different values of $\alpha$ as indicated.

sor nodes, we proposed two Markov models to assess the value of information that can be collected by a wireless sink and study the optimal collection rate by this wireless sink. The initial model assumed that the sensor nodes were unable to assess the presence or value of information. A refined model then assumed that sensor nodes were able to assess the presence of information but not the value of information. For both models, numerical examples revealed the complex interplay between battery dynamics and the value of information.

The methodology proposed can be extended in multiple directions. First, the time between collecting currently being geometrically distributed, deterministic or generally distributed intercollection times can be considered with some additional effort. In addition, as it can be expected that energy harvesting is bursty, Markovian energy harvesting can be considered as well. In this case, it can be expected that battery dynamics will have an even more profound impact on the value of information.

\section{Acknowledgements}

This research was partially funded by the Interuniversity Attraction Poles Programme initiated by the Belgian Science Policy Office. 


\section{References}

1. L. Atzori, A. Iera, G. Morabito. The internet of things: a survey. Computer Networks 54(15):2787-2805, 2010.

2. J. Gubbi, R. Buyya, S. Marusic, M. Palaniswami. Internet of things (IoT): a vision, architectural elements, and future directions. Future Generation Computer Systems 29:1645-1660, 2013.

3. I.F. Akyildiz, W. Su, Y. Sankarasubramaniam, E. Cayirci. Wireless sensor networks: a survey. Computer Networks 38(4):393-422, 2002.

4. I.F. Akyildiz, M.C. Vuran. Wireless sensor networks. Wiley, 2010.

5. H.M. Ammari, N. Gomes, M. Jacques, B. Maxim, D. Yoon. A Survey of Sensor Network Applications and Architectural Components. Ad Hoc $\&$ Sensor Wireless Networks 25(1-2):1-44, 2015.

6. H. Sundmaeker, P. Guillemin, P. Friess, S. Woelfflé (Eds). Vision and challenges for realising the internet of things, 2010.

7. V. Sachidananda, A. Khelil, N. Suri. Quality of Information in Wireless Sensor Networks: A Survey. In: Proc. of the 15th International Conference on Information Quality (ICIQ'10). pp. 193-207, 2010.

8. C. Bisdikian, L.M. Kaplan, M.B. Srivastava. On the quality and value of information in sensor networks. ACM Transactions on Sensor Networks 9(4):48, 2013.

9. E.C.-H. Ngai and P. Gunningberg. Quality-of-information-aware data collection for mobile sensor networks. Pervasive and Mobile Computing 11:203-215, 2014.

10. I. Ahmed, M.M. Butt, C. Psomas, A. Mohamed, I. Krikidis, M. Guizani. Survey on energy harvesting wireless communications: Challenges and opportunities for radio resource allocation. Computer Networks 88:234-248, 2015.

11. Z. Zhou, C. Du, L. Shu, G. Hancke, J. Niu, H. Ning. An Energy-Balanced Heuristic for Mobile Sink Scheduling in Hybrid WSNs. IEEE Transactions on Industrial Informatics 12(1):28-40, 2016.

12. Y. Bi, J. Niu, L. Sun, W. Huangfu and Y. Sun. Moving Schemes for Mobile Sinks in Wireless Sensor Networks. IEEE International Performance Computing, and Communications Conference. pp. 101-108, 2007.

13. C. Tunca and S. Isik and M. Y. Donmez, C. Ersoy Distributed Mobile Sink Routing for Wireless Sensor Networks: A Survey. IEEE Communications Surveys Tutorials16(2):877-897, 2014

14. B. Nazir and H. Hasbullah Mobile Sink based Routing Protocol (MSRP) for Prolonging Network Lifetime in Clustered Wireless Sensor Network In: Proc. of the 2010 International Conference on Computer Applications and Industrial Electronics. pp. 624-629,2010

15. Y. Gu, Y. Ji, J. Li, B. Zhao. ESWC: Efficient Scheduling for the Mobile Sink in Wireless Sensor Networks with Delay Constraint. IEEE Transactions on Parallel and Distributed Systems 24(7): 1310-1320, 2013.

16. M. Neuts. Matrix-geometric Solutions in Stochastic Models: An Algorithmic Approach. Dover, 1981.

17. A. Seyedi, B. Sikdar. Performance modelling of transmission schedulers capable of energy harvesting. In: Proc. of ICC, Cape Town, 2010.

18. A. Seyedi, B. Sikdar, Energy efficient transmission strategies for body sensor networks with energy harvesting. IEEE Transactions on Communications 58(7):21162126, 2010. 